\title{
High Efficiency Power Amplifier Based on Envelope Elimination and Restoration Technique
}

\author{
M.Vasić, O. García, J.A. Oliver, P. Alou, \\ D. Diaz, J.A. Cobos \\ Centro de Electrónica Industrial (CEI) \\ Universidad Politécnica de Madrid \\ Madrid, Spaiu \\ miroslav.vasic(a) upm.es
}

\author{
A.Gimeno, J.M.Pardo, C.Benavente, F.J.Ortega \\ Radio Engineering Group (GIRA) \\ Universidad Politécnica de Madrid \\ Madrid, Spain
}

\begin{abstract}
Due to complex envelope and phase modulation employed in modern transmitters it is necessary to use power amplifiers that have high linearity. Linear power amplifiers (classes $A, B$ and $A B$ ) are commonly used, but they suffer from low efficiency especially if the transmitted signal has high peak to average power ratio (PAPR). Kahn's technique based on envelope elimination and restoration (EER) is based on idea that high efficiency power supply (envelope amplifier) could be used to modulate the envelope of high efricient non linear power amplifiers (classes D or E). This paper presents solutions for power amplifier that performs envelope modulation and class $\mathbf{E}$ amplifier that is used as a non linear amplifier. The envelope amplifier is implemented as a multilevel converter in series with linear regulator and can provide up to $100 \mathrm{~W}$ of instantaneous power and reproduce $2 \mathrm{MHz}$ sine wave. The implemented Class E amplifier can operate at $120 \mathrm{MHz}$ with efficiency near to $85 \%$. The envelope amplifier and class $E$ amplifier have been integrated and eficiency and linearity of the implemented transmitter has been measured and presented.
\end{abstract}

Index Terms-Kahn's technique, envelope elimination and restoration, envelope amplifier, power amplifier, multilevel converter

\section{INTRODUCTION}

Due to the ultra fast development of communication technologies and their functionalities (data, voice and video transmitting), the bandwidth has becone of crucial importance. In order to increase the spectral efficiency, modem radio techniques use methods such as CDMA (Code Division Multiple Access), WCDMA (Wide Code Division Multiple Access), or EDGE (Enhanced Data rate for GSM Evolution) that use time variable envelopes. Due to the instantaneous envelope and phase modulation these systems require linear PA (class A, B or AB). Unfortunately, although they offer high linearity, these PA suffer from low efficiency. For example, a class B amplifier has efficiency of $\pi / 4$, but only in the case when it amplifies a sine wave of the maximal amplitude, without envelope modulation.

The power conversion efficiency of linear Radio Frequency PA (RF PA) is linited to less than $25 \%$ for signals with high envelope variations [1]. For example, WCDMA signals have a very high peak-to-average ratio, between $9 \mathrm{~dB}$ and $11 \mathrm{~dB}$, and it is necessary that the RF PA amplifies both high and low amplitude signals. In order to obtain a sufficiently high level of linearity, a technique known as back-off is used [1].

When the back-off is applied, the linear RF PA is supplied by voltage much higher than the signal's maximal amplitude. However, this leads to deteriorated efficiency of the transmitter. Poor system efficiency means significant power that is wasted and higher cooling requirements, bigger volume of devices and, in the case that they were battery operated much shorter autonomy. Having in mind that for the battery operated devices theirs autonony is crucial, the problem of low efficient RF PA gains on its importance.

There are a lot of techniques that are used in order to enhance the efficiency and linearity of RF PA (some of them will be explained in the next section), but two of them have often been exploited lately. One of them is called Kahn's Technique or Envelope Elimination and Restoration (EER) Technique [2]. The power supply is varied dynamically in the same way, but this time the load is not a linear PA, but a nonlinear PA. In this way, the RF PA works as a time domain multiplier, where the envelope modulation is injected to the non-linear PA. Figure 1 presents a block diagram of this technique.

The high efficiency of EER technique lies in the employed non-linear PAs (class E, D, F) that have high efficiency (theoretically $100 \%$ ) and its high efficiency power supply. Non-linear PAs are based on the idea of employing a transistor as a switching device, not as a current source, and in the way that the power losses on the transistor are very low, comparing them with the case when it works as a current source in linear classes.

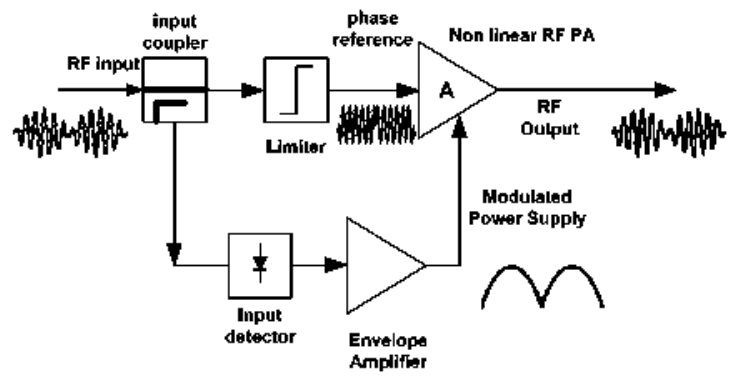

Fig.1. Block diagram of Kahn`s technique 
Thanks to the EER technique, the efficiency of the transmitter is almost constant for a wide load range and it does not depend heavily on the level of the transmitted signal, as in the case of linear amplifiers [3]. Average efficiency three to five times those of linear amplifiers has been demonstrated from $\mathrm{HF}$ to $\mathrm{L}$ band $[4,5]$.

In this paper solutions for the power supply that acts as the envelope amplifier and class E amplifier that is used as a nonlinear amplifier are presented. The envelope amplifier is based on a multilevel converter in series with a linear regulator and its bandwidth is in the range of $2 \mathrm{MHz}$, while the class E amplifier operates at $120 \mathrm{MHz}$. The instantaneous output power provided by the class $\mathrm{E}$ power amplifier is in range of $90 \mathrm{~W}$. The proposed solutions for the envelope amplifier and class E amplifier are integrated into an EER transmitter and set of tests have been conducted in order to characterize the transmitter

\section{ENVELOPE AMPLIFIER}

Due to high bandwidth requirements, conventional solutions for tracking power supplies [6-8] are not energy efficient. In [9] a buck converter is integrated on a chip. It operates at $130 \mathrm{MHz}$ and the bandwidth is $15 \mathrm{MHz}$, but the peak power was just $2.2 \mathrm{~W}$, while the average power around $1 \mathrm{~W}$. For the range of power that should be used in the desired EER PA, this solution is unacceptable as well.

The proposed envelope amplifier consists of a multilevel converter and a linear regulator in series, [10]. Its block schematic is shown in Figure 2. The multilevel converter supplies the linear regulator with discrete voltage levels that are as close as possible to the output voltage of the envelope amplifier. In this way, the power losses on the linear regulator are minimal, because they are directly proportional to the difference between its input and output voltage. However, in order to guarantee correct work of the linear regulator, the output voltage of the multilevel converter has to be higher than the output voltage of the linear regulator. Time diagrams of the multilevel and linear regulator voltage are shown in Figure 3.

By using the architecture proposed in this thesis, it is possible to take advantage of all the good characteristics of the linear regulator (high bandwidth, high linearity, etc.) and to obtain significantly higher efficiency comparing it with the solution when the linear regulator is supplied by a constant voltage. Additionally, the need for the complicated output filter design is avoided because most of the switching noise that comes from the switching converter will be filtered by the linear regulator and the feedback loop that is used can be very simple.

Thanks to the linear regulator that is employed as a post regulator, the output voltage will not have any voltage ripple, which is always present if only a switching de-de converter is used. In some applications for the envelope amplifier, the specifications for the voltage ripple, and not the desired bandwidth, lead to the necessity of very high switching frequency [7].

The main disadvantage of this architecture is that it is composed of two stages, and in order to obtain high efficiency of the envelope amplifier, it is necessary that the efficiency of both stages should be as high as possible. The efficiency of the linear amplifier will depend on the regulating precision of the dc-dc switching converter, and in order to obtain good precision for the linear regulator's input voltage the switching converter should operate at the switching frequency that is at least 5 times higher than the desired bandwidth of the envelope amplifier's output voltage [11]. If the requested bandwidth is $2 \mathrm{MHz}$, the switching frequency should be $10 \mathrm{MHz}$. At such a high switching frequency the efficiency of conventional converters (buck, boost, buck-boost etc) drops heavily due to high switching losses.

\section{A. Optimization of number of levels}

In order to optimize the efficiency of the linear regulator it is necessary to make a proper decision about the voltage levels that should be generated by the multilevel converter. The input voltage of the linear regulator is the output voltage of the multilevel converter and it is a function of the transmitted signal's envelope. It can be represented as:

$$
V_{\text {in linear regulator }}=V_{\text {in linear regulator }}\left(V_{\text {envelope }}\right)
$$

where $V_{\text {envelope }}$ is the value of the signal's envelope.

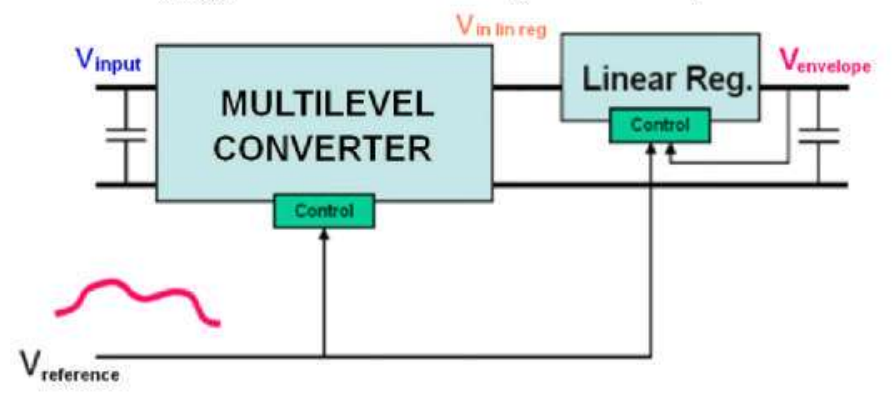

Fig.2. Simplified schematic of the proposed envelope amplifier

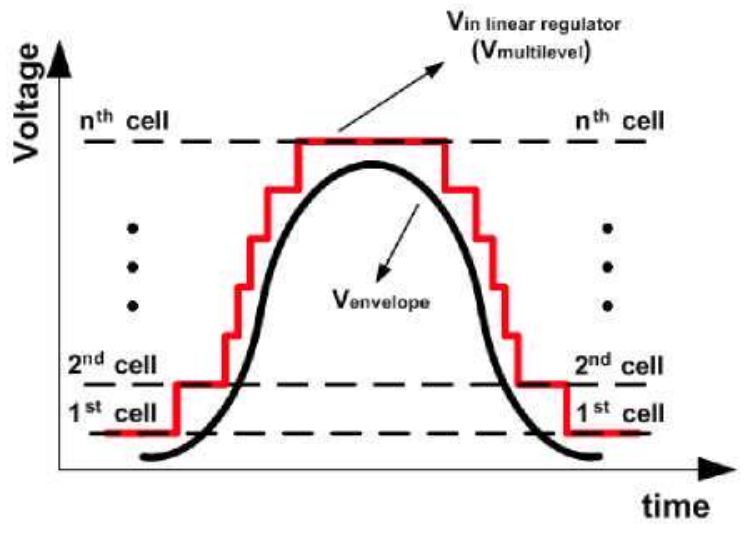

Fig.3.Time diagrams of the proposed envelope amplifier 
Thus, for the given value of the signal's envelope, the instantaneous efficiency of a linear regulator can be calculated as:

$$
\eta_{\text {instantaneous }}=\frac{V_{\text {envelope }}}{V_{\text {in linear regualtor }}\left(V_{\text {envelope }}\right)}
$$

As it is explained in [10] using equation (2) and information about the density of the probability for the signal's envelope, it is possible to calculate the average efficiency of the proposed envelope amplifier.

By selecting an appropriate distribution of the voltage levels it is possible to maximize the efficiency of the envelope amplifier for the given number of the voltage levels.

Figure 4 shows the comparison of the envelope amplifier's efficiency when the equidistant and optimized voltage levels are applied.

Although the common sense suggests employing a high number of voltage levels, it is necessary to make a trade-off between the ideal efficiency and the feasibility to implement the desired multilevel converter. The theoretical and experimental results presented in [12] show that the number of voltage levels that should be applied is between 3 and 6 . The main reason for this it that with higher number of voltage levels the amount of parasitic components, switching losses and noise is increased and that could lead to the deterioration of the quality of the output signal and overall efficiency. In the case of the multilevel converter used in this paper it has three optimized voltage levels like it is explained in $[10,12]$.

\section{B. Implementation of the multilevel converter}

An envelope amplifier with the following specifications has been built:

- Variable output voltage from $0 \mathrm{~V}$ to $23 \mathrm{~V}$

- The maximum instantaneous power is $100 \mathrm{~W}$

- The maximum frequency of the reference signal is $2 \mathrm{MHz}$

- The multilevel converter is made with three optimized voltage levels $(12 \mathrm{~V}, 18 \mathrm{~V}$ and $24 \mathrm{~V})$

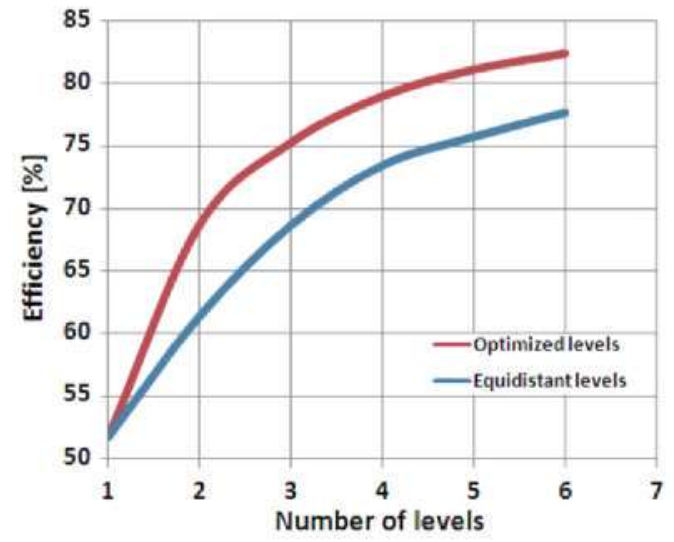

Fig.4. Comparison of the envelope's amplifier efficiencies in the case that it is implemented with the multilevel converter that reproduces optimized and equidistant voltage levels
The output voltages of the multilevel converter are selected by maximizing average efficiency of the envelope amplifier, while the MOSFET for the pass element and operational amplifier in the linear regulator have been selected to obtain high bandwidth of the envelope amplifier. The bandwidth of the selected operational amplifier (LM6172) is $100 \mathrm{MHz}$ in open loop, while the MOSFET (BLF 177) is from HF/VHF power MOS family of transistors.

The multilevel converter has been implemented using independent voltage cells that are put in series and the simplified schematic of the complete system can be seen in Figure 5.

The output voltages of the envelope amplifier and the multilevel converter for a $2 \mathrm{MHz}$ sine wave are shown in Figure 6.

The efficiency of the prototype is measured for different sine waves and the results are compared with the efficiency of an ideal linear regulator and summarized in [10].

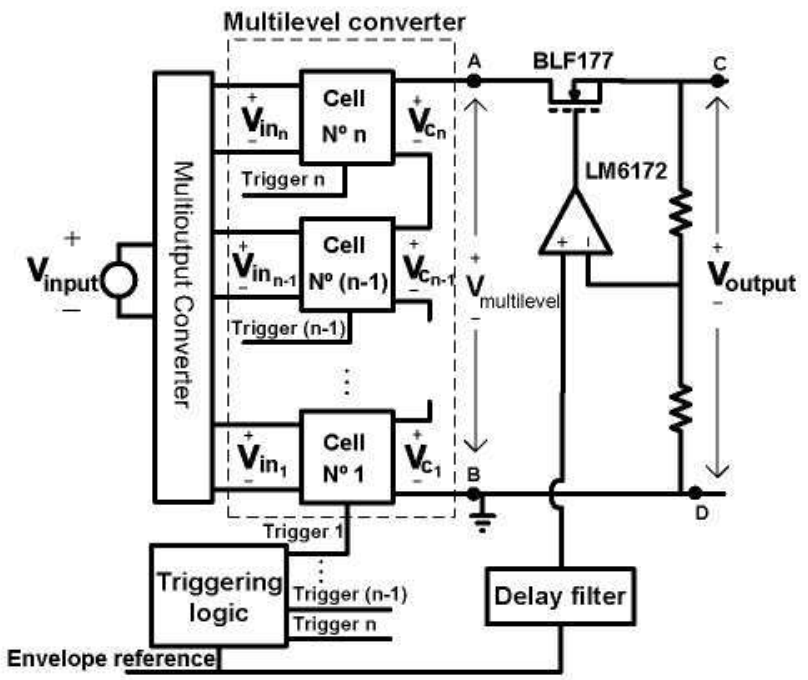

Fig.5. Simplified schematic of the implemented envelope amplifier

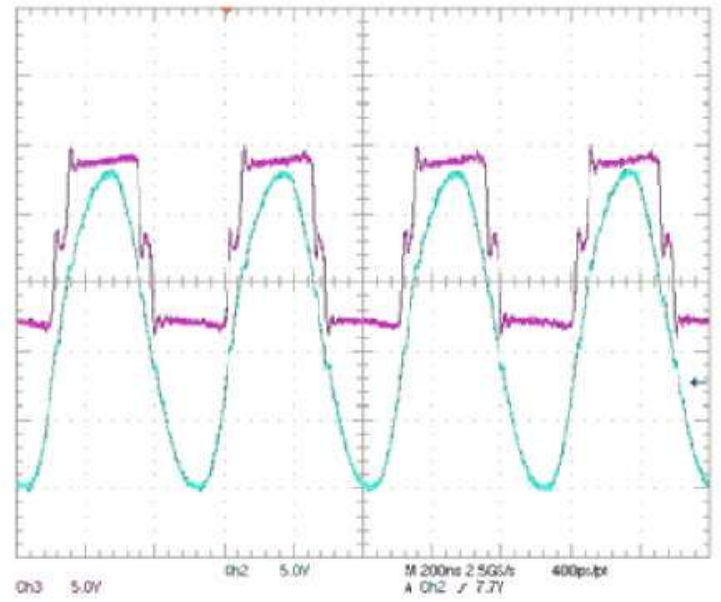

Fig.6. Output voltage of the multilevel converter and envelope amplifier when a $2 \mathrm{MHz}$ sine wave is reproduced 


\section{Class E AMPLIFIER}

The class E amplifier that is used to amplify the constant envelope, phase modulated, component of the signal, operates at the VHF band and exhibits wide fractional bandwidth (from $95 \mathrm{MHz}$ to $120 \mathrm{MHz}$ ). The drain to source voltage of the implemented class $\mathrm{E}$ amplifier can be seen in Figure 7 at $120 \mathrm{MHz}$ showing that the amplifier is operating near nominal Class-E conditions.

The design of the amplifier and its load network has been optimized to reduce power losses to a minimum. The results of efficiency measurements are shown in Figure 8. When the class $\mathrm{E}$ amplifier is supplied with constant voltage of $24 \mathrm{~V}$, its output power exhibits a peak value of $90 \mathrm{~W}$ operating between $100 \mathrm{MHz}$ and $110 \mathrm{MHz}$. The drain efficiency of the amplifier in that frequency range is around $85 \%$ (A Bird $5000 \mathrm{EX}$ wattmeter has been used to measure output power, the accuracy of this instrument is $5 \%$ ).

The RF power MOSFET used with this amplifier is a MRF6V4300N from Freescale Semiconductors. Input and output ports have been modeled for switching operation using the model proposed in [13].

This amplifier has been designed based upon the load impedance synthesis design technique as shown in [14] and simulated and optimized using Advanced Design System (ADS) software form Agilent.

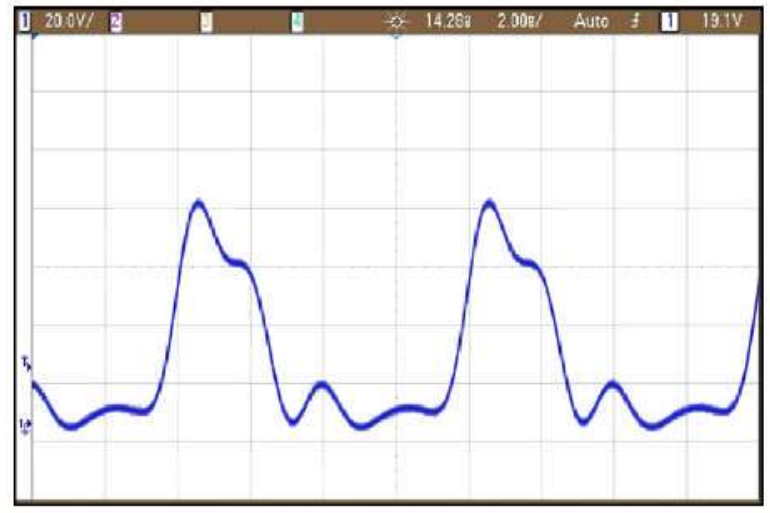

Fig.7. Drain voltage of the implemented class $\mathrm{E}$ amplifier when it operates at $120 \mathrm{MHz}$

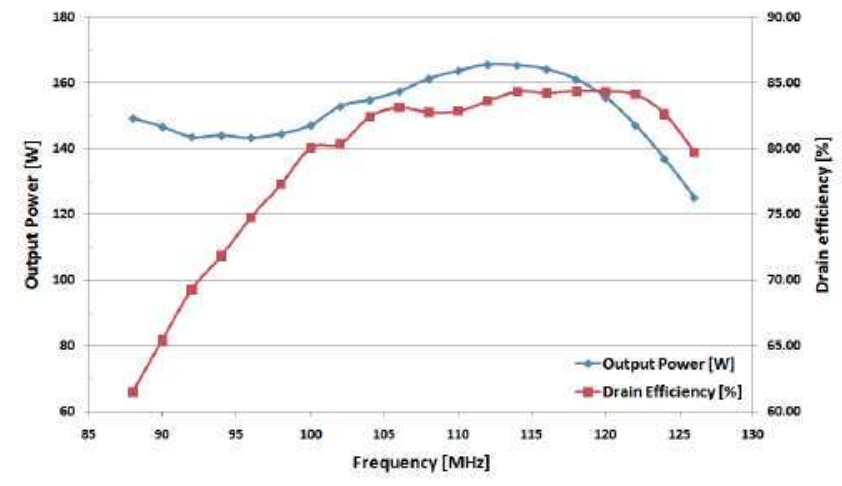

Fig 8. Measured output power and the efficiency of the implemented class $\mathrm{E}$ for different switching frequencies (instrument accuracy: 5\%).

\section{INTEGRATION OF THE ENVELOPE AMPLIFIER WITH THE CLASS E AMPLIFIER}

The integration of the implemented envelope amplifier and the class $\mathrm{E}$ amplifier has been done in order to demonstrate the advantage of one EER system over a linear power amplifiers and that overall efficiency is increased for the same level of linearity. The efficiency of an EER transmitter depends on the efficiency of the envelope amplifier and the efficiency of the nonlinear amplifier. Even more, the overall efficiency can be roughly estimated as a product of efficiencies of the en velope amplifier and nonlinear amplifier.

Besides the envelope amplifier and class $E$ amplifier, the implemented transmitter has a module that is used to receive the RF reference signal and to generate the phase and envelope references. It consists of a FPGA Virtex 4 development board fitted with $\mathrm{D} / \mathrm{A}$ and $\mathrm{A} / \mathrm{D}$ converters. The transmitter receives the $\mathrm{RF}$ reference signal through $\mathrm{A} / \mathrm{D}$ converters, and the envelope and phase references are extracted inside a FPGA. The digitalized references are converted to analog signals using high speed D/A converters, and connected to the envelope and class $\mathrm{E}$ amplifiers. Additionally, two delay filters and the envelope's amplifier triggering logic are implemented in the employed FPGA. The first delay filter is used in order to synchronize the envelope reference signal connected to the linear regulator with the output voltage of the multilevel converter in order to avoid the distortion of the reproduced envelope. The differential delay between the produced envelope and phase modulation should not be higher than one tenth of the bandwidth of the RF signal [15]. The second delay filter is used to adjust the differential delay in order to obtain high overall linearity of the system. The triggering logic needed by the multilevel converter is a simple set of comparators that has to regulate the state of the voltage cells.

Figure 9 shows the implemented EER transmitter.

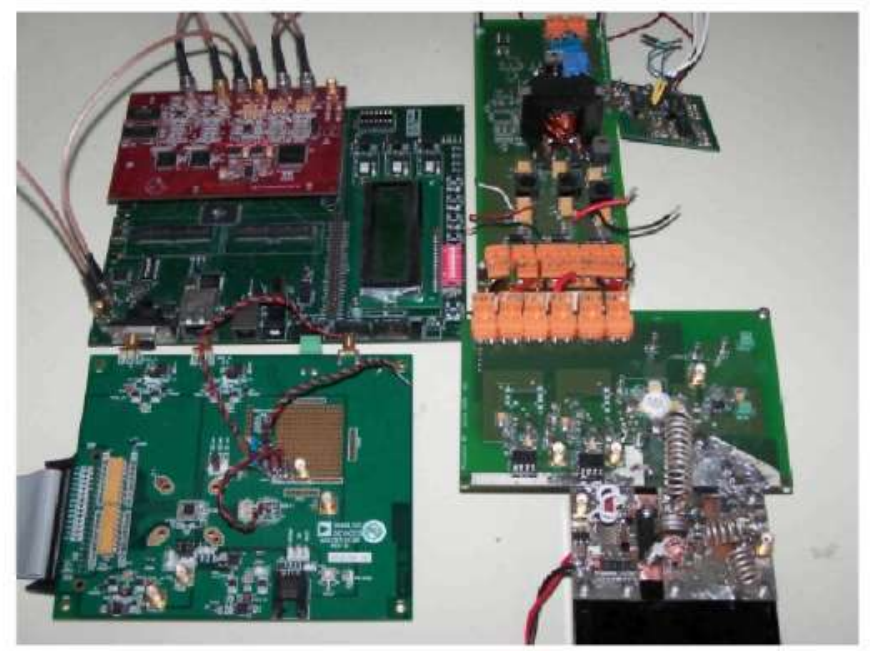

Fig.9. Photograph of the implemented EER Transmitter (on the right) with FPGA board, A/D and D/A converters (on the left) 
In [16] the results of the efficiency measurements in the case of a simple AM modulation have been presented. It has been demonstrated that the efficiency of the implemented EER PA is significantly higher than the theoretical efficiency of the class B amplifier when the signals with high PAPR (Peak to Average Power Ratio) are transmitted. The power losses were up to $40 \%$ lower in the implemented EER PA than in the case of the ideal class B PA:

In this paper the experiments regarding the linearity of the system and its efficiency with more complicated modulations are presented. The class $\mathrm{E}$ amplifier is seen as resistive $(5 \Omega)$ impedance by the implemented envelope amplifier.

\section{A. Linearity Test}

In order to measure the overall linearity of the transmitter the two tone test has been conducted. The two tone test can, actually, be presented as Double Side Band - Suppressed Carrier (DSB-SC) Modulation. A DSB-SC wave is given by:

$$
s(t)=m(t) A_{c} \cos \left(\omega_{c} t\right)
$$

where $m(t)$ is the transmitted signal, and $\omega_{\mathrm{c}}$ angular speed of the carrier.

Analyzing equation (3) it can be concluded that the spectrum of the modulated signal is actually the same as the signal's base band spectrum, just translated to high frequencies. If the transmitted signal is a simple sine wave of frequency $f_{s}$, then the envelope of the signal can be presented as:

$$
\text { envelope }_{\text {ref }}=\left|\sin \left(2 \pi f_{s} t\right)\right|
$$

and the output signal is then:

$$
s(t)=\left|\sin \left(2 \pi f_{s} t\right)\right| \cos \left(2 \pi f_{c} t+\varphi_{s}\left(2 \pi f_{s} t\right)\right)
$$

Therefore, from equations (3)-(5) it yields that the reference signal for the class $\mathrm{E}$ amplifier is a sine wave with the same frequency as the carrier and that this sine wave has to change its phase for 180 degrees each time when the envelope reference is equal to zero.

These two reference signals are created in MATLAB and then stored in a look-up table in an FPGA. In Figure 10 the envelope reference, the output of the multilevel converter, the output of the envelope amplifier and the output of the implemented EER transmitter are shown during the linearity measurements. Figure 11 shows the spectrum of the output of the transmitter.

In Figure 10 a huge amount of the RF noise in the envelope reference can be seen but, due to the low pass property of the implemented envelope amplifier, that noise does not have influence on the output signal of the envelope amplifier. The spectrum of the transmitter's output shows two harmonics around $125 \mathrm{MHz}$, like it is expected, but there are more components that are products of AM-PM distortion of the class $\mathrm{E}$ amplifier. It can be seen that the attenuation of the highest additional harmonic is $18 \mathrm{~dB}$, which means that the quality of the output signal will not be high.

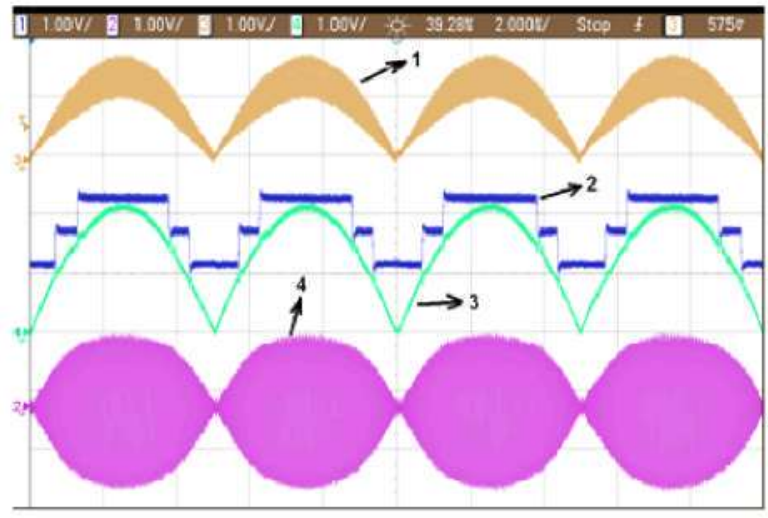

Fig10. The reference signal (label 1, channel 3), the output of the multilevel converter (label 2, channel 4), the output of the envelope amplifier (label 3, channel 1) and the output of the EER transmitter (label 4, channel 2) when the envelope reference signal is rectified $100 \mathrm{kHz}$ sine wave

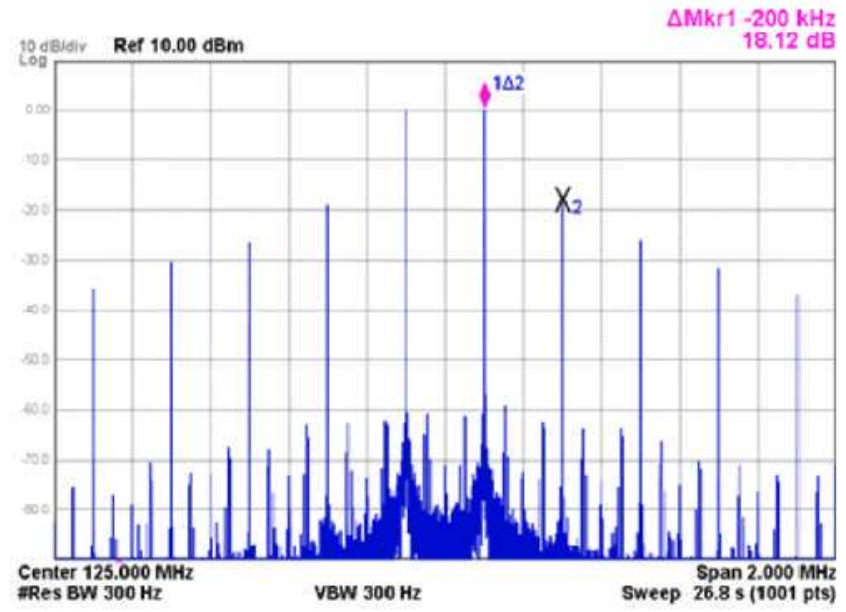

Fig.11. Spectrum of the output of the EER transmitter when the transmitted signal is a sine wave of $100 \mathrm{kHz}$, carrier is a $125 \mathrm{MHz}$ sine wave and the applied modulation is DSB-SC

The AM-PM conversion is due to the presence of the nonlinear capacitor between the drain and the source of the class E MOSFET. Depending on the applied supply voltage, this capacitor changes its value and it influences the phase delay between the signal sent to the MOSFET's gate and the signal reproduced by the class $\mathrm{E}$ amplifier.

In order to improve this attenuation, a measurement of the AM-PM conversion has been conducted and it is represented in the terms of the aforementioned phase delay. The result of the measurement can be seen in Figure 12.

The predistorter used to enhance the linearity is implemented in the way that it inserts the inverted phase delay in order to cancel the influence of the class E amplifier. The simplified block schematic is presented in Figure 13.

Having in mind that the relationship between phase delay and the class E amplifier supply voltage (this voltage is proportional to the envelope of the transmitted signal) is known, the predistorter is made as a look-up table. Each time that the envelope reference is sent, the corresponding phase delay is inserted in the phase modulation. 


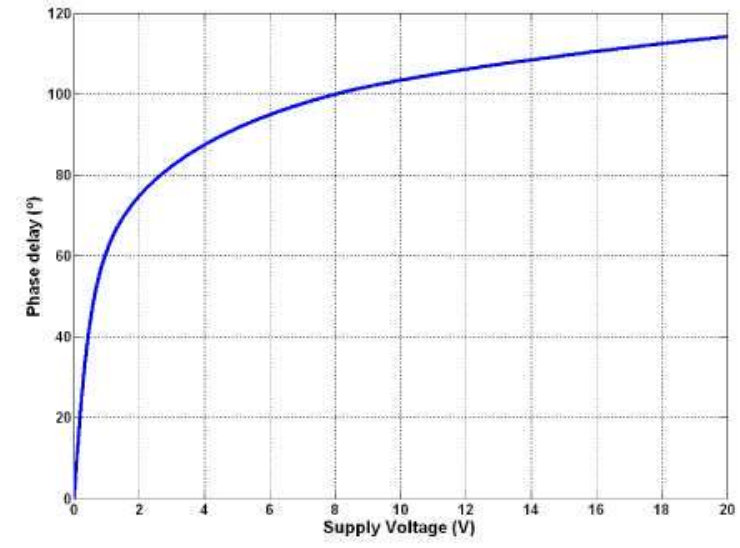

Fig. 12. Dependency of the phase delay between the output of the implemented class E PA and gate signal depending on the supply voltage of the PA

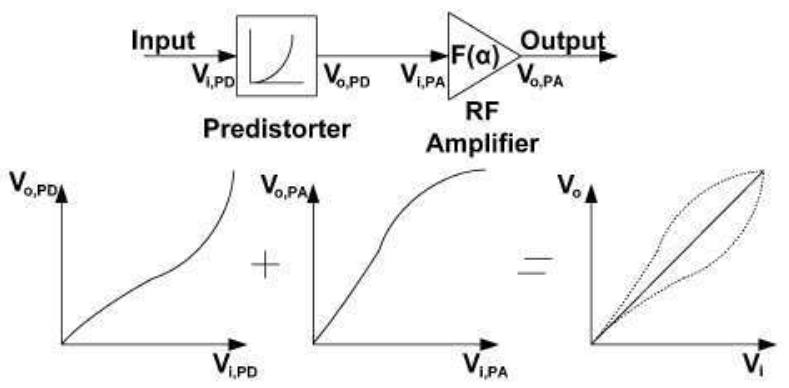

Fig. 13. Block diagram of predistortion concept and amplitude correction by predistortion

In order to facilitate the testing, the two-tone signal is generated in MATLAB, and the envelope reference and predistorted phase reference are sent to the EER amplifier via the signal generator (Agilent $81150 \mathrm{~A}$ ). The measured linearity after the predistortion in the case that the $f_{s}$ is $500 \mathrm{kHz}$, and $f_{c}$ is $100 \mathrm{MHz}$.is presented in Figure 14

Figure 15 shows the attenuation of the $3^{\text {rd }}$ order intermodulation products for different carrier and and envelope frequencies.

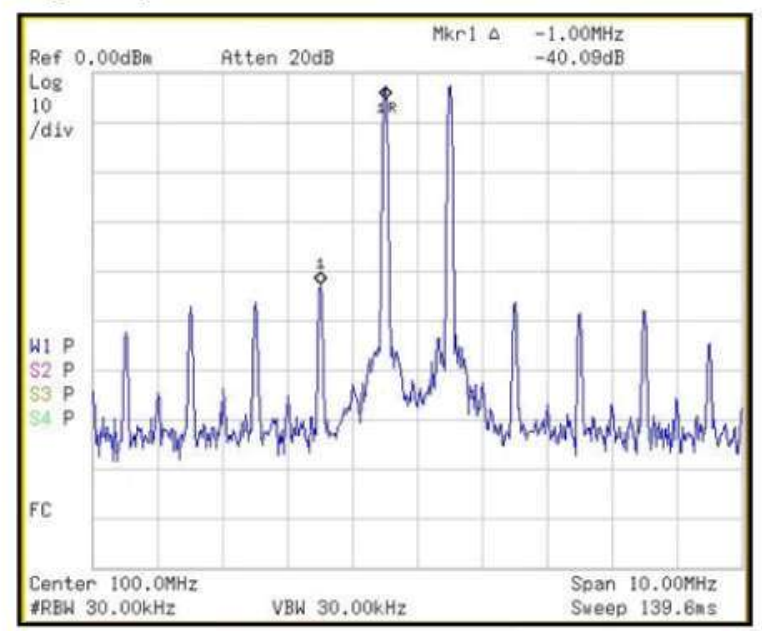

Fig.14. Spectrum of the PA's output voltage when two tones are sent as the PA's reference signal $\left(\mathrm{f}_{\mathrm{c}}=100 \mathrm{MHz}, \mathrm{f}_{\mathrm{s}}=500 \mathrm{kHz}\right)$

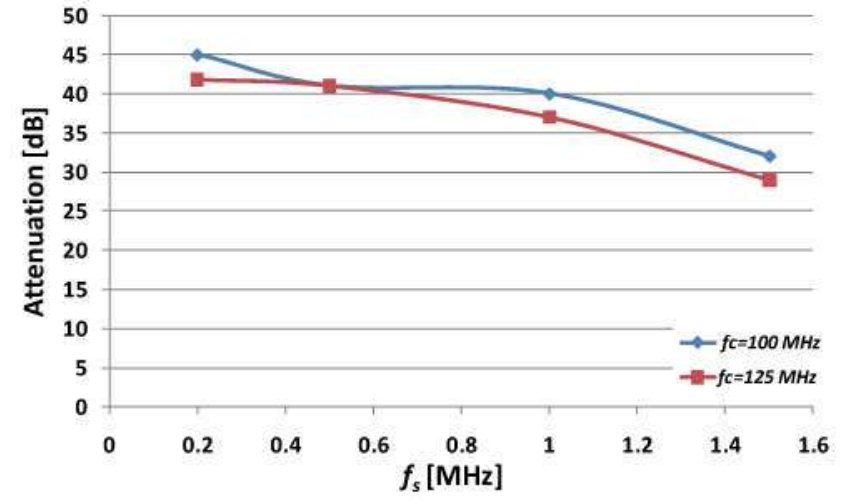

Fig. 15. Measured attenuation of the third order intermodulation products, for the different frequencies of the envelope $\left(\mathrm{f}_{\mathrm{s}}\right)$ and carrier signal $\left(\mathrm{f}_{\mathrm{c}}\right)$

\section{B. Efficiency measurements}

Once the high linearity of the PA has been obtained, efficiency measurements in the case of a 64QAM have been performed.

The 64QAM-modulated signal can be presented as:

$$
\begin{aligned}
& x(t)=Q(t) \sin (\omega t)+I(t) \cos (\omega t) \\
& Q(t), I(t) \in[-7,-5,-3, \ldots, 3,5,7]
\end{aligned}
$$

Graphically, the 64QAM modulation can be presented with its constellation as in Figure 16.

In order to make the test as real as possible, the I and Q signals are generated as random signals with Gaussian distribution. In that way, the envelope of the test signal has Rayleigh's distribution with a high PAPR. Figure 17 shows the constellation and the transitions of the generated signal.

As it can be seen, due to the Gaussian distribution of the I an Q signals, the probability that I and $\mathrm{Q}$ have high values at the same time (and in that way high envelope values) is low, and it correspond to the high PAPR signal.

The impedance seen by the envelope amplifier is around $5 \Omega$ and the maximal value of the envelope is $22 \mathrm{~V}$. This leads to the conclusion that the envelope amplifier has to provide up to $100 \mathrm{~W}$ of instantaneous power.

The generated signal has a ratio between the maximal value and the average value of $8.6 \mathrm{~dB}$. The density of the probability for the envelope is shown in Figure 18. High PAPR can be observed once again. The high peak that is presented for the envelope value of $2 \mathrm{~V}$ is due to the short interval of time where the envelope has this value in order to facility the synchronization between envelope and phase modulation. However, that part of the envelope is just $3 \%$ of the overall signal and it does not have any significant influence on the overall efficiency.

The same I, Q pattern is used with different data rates in order to see how the bandwidth of the transmitted signals influences the efficiency of the implemented EER transmitter. The bandwidth of the transmitted signal is, approximately, twice the bit rate. 


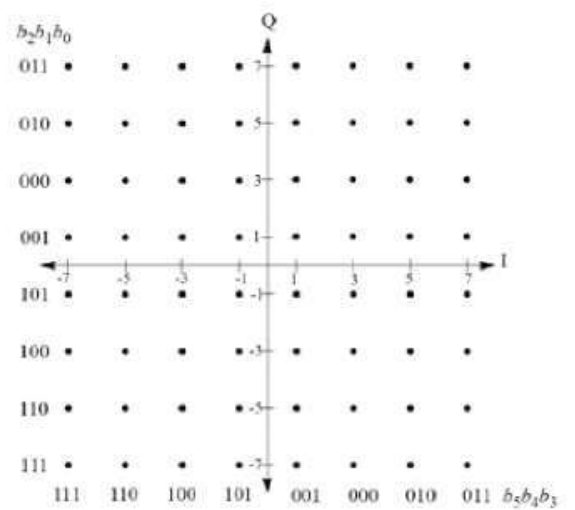

Fig. 16. Constellation of the $I$ and $Q$ signals for 64QAM modulation

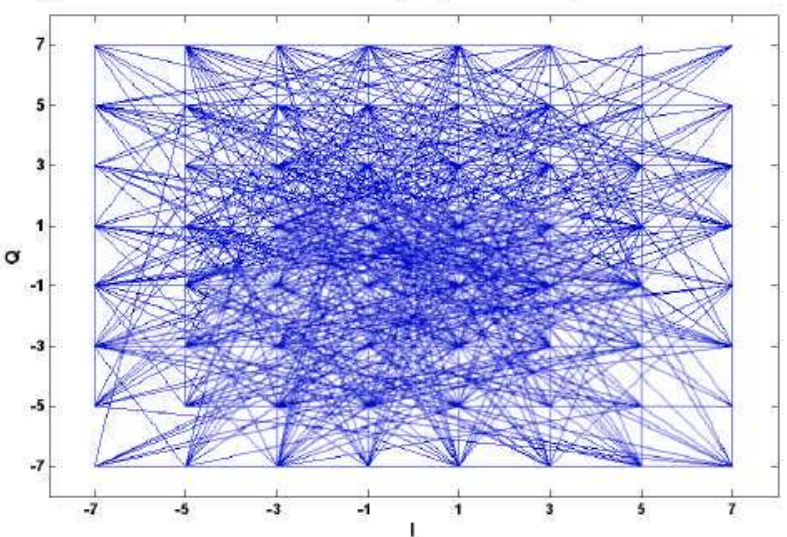

Fig.17. Generated constellation and transitions of the $I$ and $Q$ signal in the case of 64QAM modulation

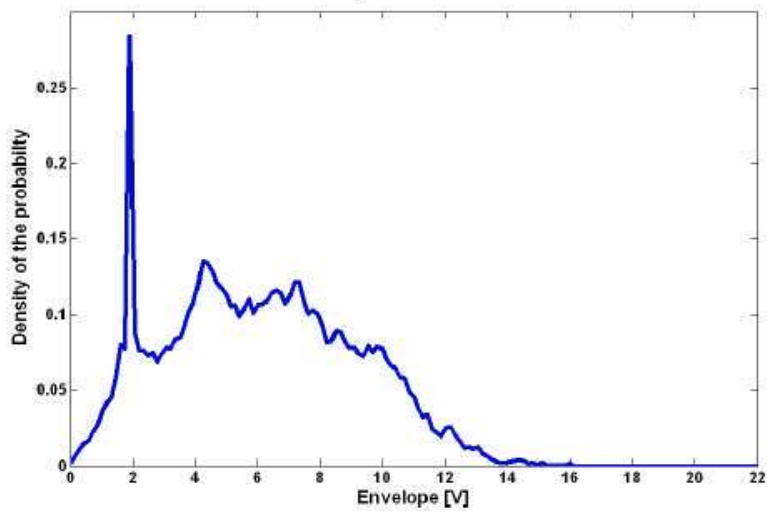

Fig.18. Density of the probability of the envelope signal for the generated test pattern based on 64QAM modulation

The measured efficiency is presented in Figure 19 and it is compared with the theoretical efficiency of an ideal class B amplifier supplied by $24 \mathrm{~V}$. The efficiency measurements are conducted with carrier signals of $100 \mathrm{MHz}$ and $125 \mathrm{MHz}$. The reason for this is to see how the efficiency of the system depends on the frequency of the carrier signal and, in that way, on the input impedance of the class $\mathrm{E}$ amplifier. The average output power of the transmitted signal is $13 \mathrm{~W}$.

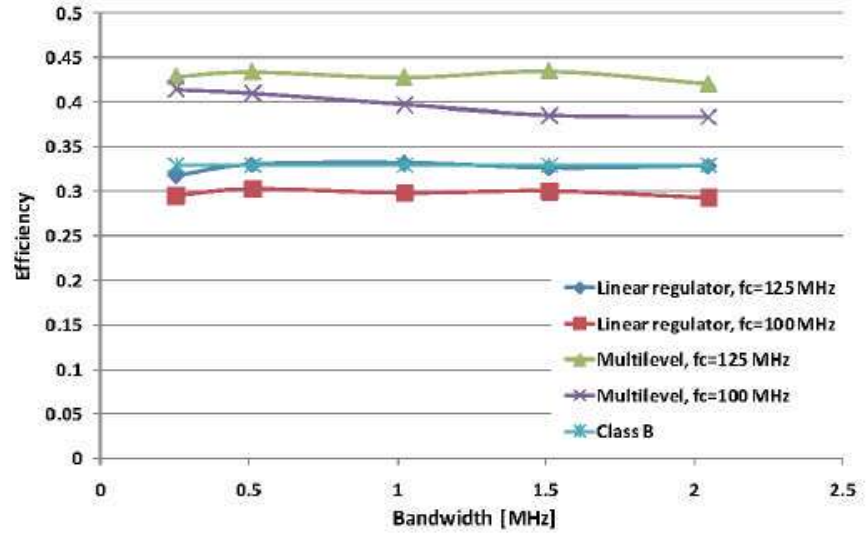

Fig.19. Measured efficiency of the implemented EER PA in the case when the class $\mathrm{E}$ amplifier is supplied by classical linear regulator supplied by constant voltage and supplied by the proposed hybrid solution. The measured efficiency is compared with the theoretical efficiency of an ideal class B PA supplied by $24 \mathrm{~V}$

When the carrier signal is $125 \mathrm{MHz}$, it can be seen that if the implemented EER PA uses a linear regulator supplied by a constant voltage as the envelope amplifier the efficiency is practically the same as the theoretical efficiency of the ideal class B amplifier. In the case that the multilevel converter is used the efficiency is improved from approximately $32 \%$ to $43 \%$ (the power losses are decreased for $25 \%$ ). The main reason for this is the high $\mathrm{PAPR}$ of the transmitted signal.

For the carrier signal of $100 \mathrm{MHz}$ similar conclusions can be made. The implemented EER PA exhibits higher efficiency if it employs the multilevel converter in series with linear regulator than the efficiency when only the linear regulator is used. The efficiency is improved from $30 \%$ to $40 \%$ (improvement of $25 \%$ in the terms of power losses) and it is higher than the estimated efficiency of ideal class B amplifier for the same signal pattern. However, when the frequency of the carrier is $100 \mathrm{MHz}$, the input impedance of the class $\mathrm{E}$ amplifier is lower than in the case of $125 \mathrm{MHz}$ carrier wave. Due to this, the envelope amplifier has to process higher currents and it has more power losses. Therefore, the overall efficiency is slightly lower for the 100 $\mathrm{MHz}$ carrier than in the case when the frequency of the carrier is $125 \mathrm{MHz}$.

The time waveforms of the multilevel converter, envelope amplifier and output of the implemented EER PA in the case that the transmitted signal has the bandwidth of $2 \mathrm{MHz}$ can be observed in Figure 20. The constellation and the spectrum of the demodulated signal can be seen in Figure 21. The demodulation is performed by Agilent MxA Signal Analizer N9020A.

By analyzing the constellation of the demodulated signal, it can be concluded that the EER PA has correctly reproduced the signal because there can be clearly distinguished all 64 possible points. The error vector magnitude (the measure of how far the points are from the ideal locations) is around $2.7 \%$. 


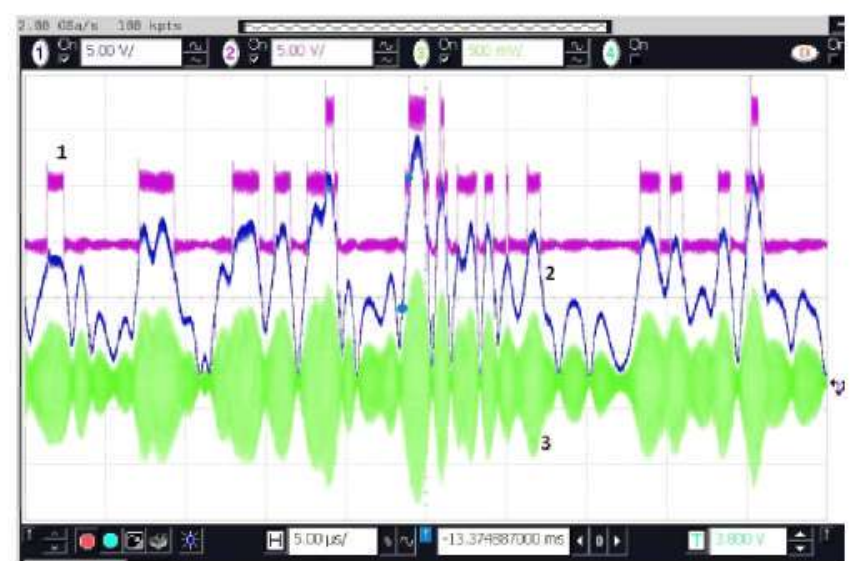

Fig.20. Oscilloscope capture of the multilevel converter's output voltage (label 1), envelope amplifier's output voltage (label 2) and complete RF signal (label 3) in the case when a 64QAM signal with the bit rate of $1024 \mathrm{kbit} / \mathrm{s}$ is amplified

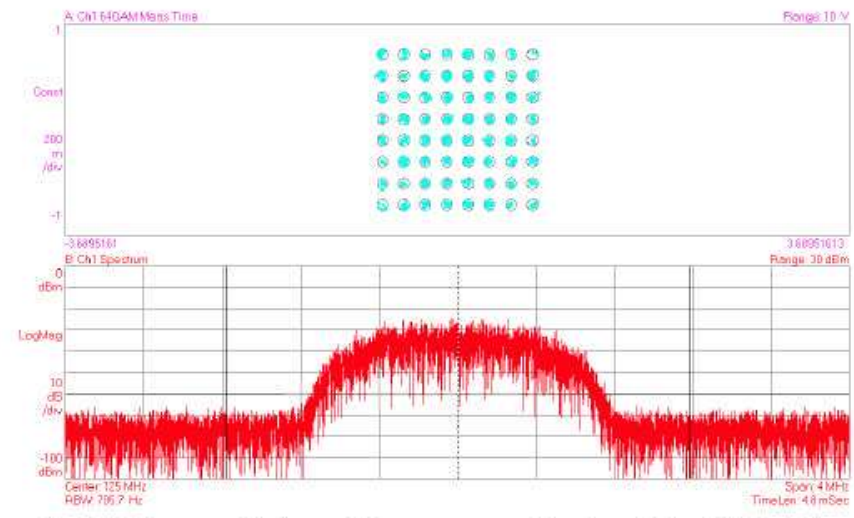

Fig.21. The constellation and the spectrum of the demodulated 64QAM signal amplified by the implemented EER PA

\section{V.CONCLUSIONS}

In this paper a radio transmitter based on the Kahn's technique (EER) is presented. The implemented transmitter is composed of envelope amplifier (responsible for envelope injection) and a class-E amplifier (performs phase modulation). The envelope amplifier has to fulfill strict requirements regarding its bandwidth and linearity, and, therefore, it is implemented as a multilevel converter in series with a linear regulator. In this way, high frequency signals can be reproduced applying relatively low switching frequency.

The implemented EER PA can provide up to $100 \mathrm{~W}$ of instantaneous power and reproduce a $2 \mathrm{MHz}$ sine wave of maximal excursion (from $0 \mathrm{~V}$ to $22 \mathrm{~V}$ ).

The linearity of the implemented EER PA was measured using two tone signals. First linearity measurements indicated that due to the non-linear effects in the class $\mathrm{E}$ amplifier, it would be necessary to use predistortion in order to increase the system's linearity. By employing predistortion, the attenuation of the intermodulation products of the third order is around $40 \mathrm{~dB}$ in wide range of bandwidths (up to $1 \mathrm{MHz}$ ).
Once the system's high linearity was obtained, the complete EER PA was tested with a 64QAM modulation where the envelope of the signal has high PAPR $(8.6 \mathrm{~dB})$. By measuring the power consumption it was demonstrated that the power losses of the implemented EER PA are, approximately, 25\% lower than the power losses of an ideal linear class B PA. The maximum bandwidth of the transmitted signal was around $2 \mathrm{MHz}$ (bit rate was 1024 $\mathrm{kbit} / \mathrm{s})$.

\section{REFERENCES}

[1] N.D.Lopez, X.Jiang, D. Maksimovic, Z.Popovic, "A High-Efficiency Linear Polar Transmitter for EDGE", IEEE Radio and Wireless Symposium Digest, January 2008, pages 199-202

[2] T.Oshima, M.Kokubo, "Simple polar-loop transmitter for dual-mode Bluetooth", Proceedings on IEEE International Symposium on Circuits and Systems, ISCAS 2005, Volume 4, May 2005, pages:3966-3969

[3] F.H.Raab; P. Asbeck, S. Cripps, P.B. Kenington, Z.B. Popovic,N. Pothecary, J.F. Sevic, N.O. Sokal, "Power amplifiers and transmitters for RF and microwave",IEEE Transactions on Microwave Theory and Techniques, Volume 50, Issue 3, March 2002 Page(s):814-826

[4] F. H. Raab and D. J. Rupp, "High efficiency single-sideband HF/ VHF transmitter based upon envelope elimination and restoration," Proc. Sixth Int. Conf. HF Radio Systems and Techniques (HF '94) (IEE CP 392), York, UK, pp. 21-25, July 4 - 7, 1994.

[5] F. H. Raab, B. E. Sigmon, R. G. Myers, and R. M. Jackson, "L-band transmitter using Kahn EER technique," IEEE Trans. Microwave Theory Tech., pt. 2, vol. 46, no. 12, pp. 2220-2225, Dec. 1998.

[6] J. Staudinger, B. Gilsdorf, D. Newman, G. Norris, G. Sadowniczak, R. Sherman, T. Quach, "High efficiency CDMA RF power amplifier using dynamic envelope tracking technique," Microwave Symposium Digest., IEEE MTT-S International, Vol. 2, June 2000, Pages: 873-876

[7] M. Hoyerby, M. Andersen, "Ultrafast Tracking Power Supply with Fourth-Order Output Filter and Fixed-Frequency Hysteretic Control", IEEE Trans. on Power Electronics, Volume: 23, Issue: 5, 2008, Pages: 2387-2398

[8] A. Soto, J.A. Oliver, J.A. Cobos, J. Cezon, F. Arevalo, 'Power supply for a radio transmitter with modulated supply voltage", Applied Power Electronics Conf., APEC '04, Volume: 1, Feb. 2004 Pages:392 - 398

[9] V. Pinon, F. Hasbani, A. Giry, D. Pache, C. Gamier, "A Single-Chip WCDMA Envelope Reconstruction LDMOS PA with $130 \mathrm{MHz}$ Switched-Mode Power Supply," Solid-State Circuits Conference, 2008. ISSCC 2008.pp.564-636, 3-7 Feb. 2008

[10] M.Vasić, O.Garcia, J.A.Oliver, P.Alou, D.Diaz, J.A.Cobos, "Multilevel Power Supply for High Efficiency RF Amplifier", IEEE Transactions on Power Electronics, Volume 25, Issue 4, pages 1078 - 1089, 2010.

[11] L.Marco, E. Alarcon, D. Maksimovic, "Effects of switching power converter nonidealities in Envelope Elimination and Restoration technique", IEEE International Symposium on Circuits and Systems, ISCAS 2006, 21-24 May 2006.

[12] M.Vasic, "Wide Bandwidth High Efficiency Power Converter for RF Amplifiers", $\mathrm{PhD}$ Thesis, Universidad Politcenica de Madrid, Spain, June 2010.

[13] N. O. Sokal, R. Redl, "Power Transistor Output Port Model", RF Design, Vol. 10, No. 6, pp. 45-48, 50, 51, 53, June 1987.

[14] Ortega-Gonzalez,F.J, "Load-Pull Wideband Class-E Amplifier", Microwave and Wireless Components Letters, Vol. 17, No. 3, pp. 235237, March 2007

[15] F.H. Raab, "Intermodulation Distortion in Kahn-Technique Transmitters", IEEE Transactions on Microwave Theory and Techniques, Volume 44, Issue 12, Part 1, Decemeber 1996, Pages: 2273-2278

[16] M. Vasić; O. Garcia, J.A. Oliver, P. Alou, D. Diaz, J.A. Cobos, "High Efficiency Power Amplifier for High Frequency Radio Transmitters", Applied Power Electronics Conference and Exsposition, APEC 2010, February 2010 